\title{
Assessment of Myocardial Mechanics in Acute Rheumatic Fever Using Speckle Tracking Echocardiography
}

\author{
Utku Pamuk $^{1}$, Hazım Gursu ${ }^{2}$, Emine $\mathrm{Azak}^{2}$, and İlker Çetin ${ }^{2}$ \\ ${ }^{1}$ University of Health Sciences, Ankara Child Health and Diseases Hematology Oncology \\ Training and Research Hospital \\ ${ }^{2}$ University of Health Sciences, Ankara City Hospital
}

May 30, 2021

\begin{abstract}
Objectives: This study aims to evaluate the role of speckle tracking echocardiography (STE) to identify myocardial deformation abnormalities in acute rheumatic fever. Methods: Twenty-seven patients and twenty-seven healthy children were studied prospectively. The patients were divided into two subgroups as moderate/severe carditis and mild/no carditis according to valve involvement. The left ventricular global longitudinal strain (LVGLS) and strain rate (LVGLSR), left ventricular global circumferential strain (LVGCS) and strain rate (LVGCSR), and right ventricular global longitudinal strain (RVGLS) and strain rate (RVGLSR) were examined by STE. Results: Left ventricular global longitudinal strain, LVGLSR, LVGCS, LVGCSR, RVGLS and RVGLSR were significantly lower in patients in acute phase of disease than controls. There were no differences in strain and strain rates between patient subgroups before treatment. After acute phase, statistically significant improvements were found in LVGLS, LVGLSR, LVGCSR values of the patients with moderate/severe carditis and LVGLS, LVGCS, LVGCSR, RVGLS, RVGLSR values of the patients with mild/no carditis. The strain parameters of the patients and controls did not differ significantly after the treatment. Conclusions: In acute phase of rheumatic heart disease, patients have reduced left and right ventricular strain and strain rates, which shows improvements after treatment.
\end{abstract}

\section{Introduction}

Rheumatic heart disease (RHD) is endemic in developing countries specifically among school-age children. Joint, skin and central nervous systems might be involved but heart involvement, which can cause mortality and morbidity, is the most important clinical outcome. Heart involvement is thought to start from the endocardium and progress through the pericardium [1]. Another opinion that has been put forward is that patients diagnosed with RHD can have some degree of myocardial involvement in general [2]. In patients with cardiac involvement, the degree and severity of valve involvement can be diagnosed easily, however it is difficult to identify myocardial involvement and its severity. When there is pericardial involvement, the assumption is that there is myocardial involvement as well. In the absence of pericardial involvement, it is difficult to diagnose myocardial involvement even with endomyocardial biopsy [3]. To identify such involvement more advanced diagnostic techniques such as speckle tracking echocardiography (STE) need to be utilized.

Speckle tracking echocardiography has been shown to be more effective than the conventional measures of ejection fraction (EF) and shortening fraction (FS) in evaluating heart functions [4]. Many patients having heart failure, hypertension, Systemic Lupus Erythematosus, Duchenne type muscular dystrophy who have been evaluated as normal with conventional techniques, were identified to have abnormalities in strain values [5-8]. 
In this study, we tried to demonstrate that during the acute stages of rheumatic fever there might be abnormalities due to myocardial involvement that can be diagnosed with myocardial strain and that these abnormalities might differ in patients having mild and moderate to severe carditis and that such abnormalities might disappear after the treatment period has ended.

\section{Materials and Methods}

\section{Study population}

Twenty-nine patients (mean age 12.3 \pm 3.2 years, range 6-18 years; 14 male/15 female) diagnosed with acute rheumatic fever from January 2016 to January 2017 were prospectively enrolled in this study. Two patients who were diagnosed with juvenile idiopathic arthritis and systemic lupus erythematosus upon enrollment in the study were excluded afterward. The study cohort was divided into two subgroups on the basis of valve involvement [9]. The moderate/severe carditis group consisted of 12 patients with moderate/severe valvular involvement. No/mild carditis group consisted of 15 patients with mild valvular involvement or no valvular involvement. All measures were compared with values of 27 healthy children of similar sex and age (mean age $11.7 \pm 3.1$ years, range $7-17$ years; 16 male/ 11 female, $\mathrm{p}=0.781$ for age, $\mathrm{p}=0.722$ for sex).

The patients were included in the study according to previously defined revised Jones criteria for acute rheumatic fever [10]. Patients with recurrent RHD were not included in the study. Erythrocyte sedimentation rate and C-Reactive protein values were measured in all patients before and after the treatment. After a single dose of benzathine penicillin patients who were diagnosed with moderate/severe carditis were treated with corticosteroids for 4-6 weeks and after steroids, they were administered with non-steroid anti-inflammatory drugs (NSAID) for an additional 2-4 weeks. Patients who were diagnosed as no/mild carditis were treated with NSAID for 2-6 weeks after a single dose of benzathine penicillin. Both the patients and the controls underwent a standard transthoracic echocardiographic examination and STE.

The study complied with the Declaration of Helsinki and the Clinical Research Ethical Committee approved the study (decree no: 2017-027). The families of the patients provided their informed consent.

\section{Echocardiographic examination}

Echocardiography was performed by using the Philips iE33 ultrasound system (Philips, The Netherlands) and a $5 \mathrm{Mhz}$ transducer. Conventional echocardiography and STE were performed before and after treatment (4-6 weeks after-when acute phase reactants decrease). Systolic function was assessed by M-mode derived FS and EF. Left ventricular EF $>54 \%$ and $\mathrm{FS}>28 \%$ were accepted as normal left ventricular systolic function [11].

For STE, two-dimensional four-chamber images together with short-axis mid-circumferential images of the left ventricle were obtained. Three cardiac cycles were recorded as cine loop clips. Strain and strain rate measurements were performed using the software package QLAB Advanced Quantification Software (version 6.0, Philips, The Netherlands). Main measures were left ventricular global longitudinal strain (LVGLS) and left ventricular global longitudinal strain rate (LVGLSR), left ventricular global circumferential strain (LVGCS) and left ventricular global circumferential strain rate (LVGCSR), right ventricular global longitudinal strain (RVGLS) and right ventricular global longitudinal strain rate (RVGLSR). All the images were obtained in the left lateral decubitus position under ECG monitoring. All echocardiographic parameters were analyzed by one researcher at two different time points.

\section{Statistical analysis}

Study data were analyzed with the SPSS for Windows 17.0 package program (SPSS Inc., Chicago, IL). For descriptive measures, mean and standard deviation (SD) were used. The variables were considered to be distributed regularly by Shapiro-Wilk Test and comparisons between groups were made by Independent Samples T-test, one way ANOVA. The differences in each group before and after the treatment were identified by paired samples T-test. A p-value of $<0.05$ was considered as being statically significant.

\section{Results}




\section{Patient characteristics}

Fourteen of the patients enrolled in the study were male (51.9\%) while 13 were female (48.1\%). The mean age of the patients was $12.3 \pm 3.2(6-18)$ years. The hospitalization period of the patients was a median of 7 days. Polyarthralgia was the most common complaint (17 patients), followed by arthritis (10 patients) and fever ( 5 patients). We only had one patient with subcutaneous nodules. There was no patient with the diagnosis of erythema marginatum.

\section{M-mode derived echocardiographic findings}

All patients (carditis group EF $69.6 \pm 2.29 \%$, FS $38.9 \pm 1.8 \%$; mild/no carditis group EF $69.6 \pm 3.7 \%$, FS $38.99 \pm 3.2 \%$ ) and healthy controls (EF $69 \pm 2.72 \%$, FS $38.2 \pm 1.98 \%$ ) had normal systolic functions and there was no statistically significant difference between the two groups (Table 1).

Table 1. Ejection fraction (EF), Fractional shortening (FS) of the patient and control groups before treatment

\begin{tabular}{lll}
\hline & Mean EF (\%) & $p$ value \\
Moderate/severe carditis & $69.6 \pm 2.29$ & 0.788 \\
Mild/no carditis & $69.6 \pm 3.7$ & \\
Control & $69 \pm 2.72$ & \\
& Mean FS (\%) & \\
Moderate/severe carditis & $38.9 \pm 1.8$ & 0.593 \\
Mild/no carditis & $38.99 \pm 3.2$ & \\
Control & $38.2 \pm 1.98$ & \\
\hline
\end{tabular}

Furthermore, there was no statistically significant differences for EF and FS between pre-treatment (mean EF $69.3 \pm 3 \%$, FS $38.6 \pm 2.4 \%$ ) and post-treatment (mean EF $69.6 \pm 2.8 \%$, FS $38.8 \pm 2.3 \%$ ) measurements in the study group ( $\mathrm{p}=0.768$ for $\mathrm{EF}, \mathrm{p}=0.698$ for $\mathrm{FS}$ ), in moderate/severe carditis group $(\mathrm{p}=0.59$ for $\mathrm{EF}, \mathrm{p}=0.29$ for FS) and mild/no carditis group ( $\mathrm{p}=0.922$ for $\mathrm{EF}, \mathrm{p}=0.748$ for FS).

\section{Speckle tracking echocardiographic findings}

Left ventricular global longitudinal strain, LVGLSR, LVGCS, LVGCSR, RVGLS and RVGLSR were significantly lower in all the patients and the controls before the treatment. Moreover, there were no statistically significant differences for all the strain levels between moderate/severe carditis and mild/no carditis groups (Table 2).

Table 2. Strain parameters before treatment.

\begin{tabular}{|c|c|c|c|c|c|c|c|}
\hline & $\begin{array}{l}\text { Moderate/s } \\
\text { Carditis (n } \\
=12)\end{array}$ & $\begin{array}{l}\text { rAild } / \mathrm{no} \\
\text { carditis }(\mathrm{n}= \\
15)\end{array}$ & $\begin{array}{l}\text { Control (n } \\
=27)\end{array}$ & $P 1$ & P2 & P3 & $P_{4}$ \\
\hline LVGLS & $18.8 \pm 1.9$ & $18.6 \pm 2.7$ & $22.1 \pm 3.5$ & .000 & 0.007 & 0.004 & .833 \\
\hline LVGLSR & $0.3 \pm 0.2$ & $0.38 \pm 0.18$ & $0.6 \pm 0.4$ & .002 & 0.013 & 0.045 & .282 \\
\hline LVGCS & $17.9 \pm 2.5$ & $18.7 \pm 2.9$ & $21.1 \pm 3.2$ & .003 & 0.018 & 0.063 & .477 \\
\hline LVGCSR & $0.43 \pm 0.26$ & $0.33 \pm 0.22$ & $0.68 \pm 0.35$ & .001 & 0.098 & 0.002 & .325 \\
\hline RVGLS & $18.2 \pm 2.9$ & $19.8 \pm 3.1$ & $23.9 \pm 6.8$ & .000 & 0.009 & 0.060 & .193 \\
\hline RVGLSR & $0.38 \pm 0.29$ & $0.20 \pm 0.14$ & $0.7 \pm 0.42$ & .001 & 0.025 & 0.061 & .197 \\
\hline
\end{tabular}

P1: SC/MNC/C, P2: SC/C, P3: MNC/C, P4: SC/MNC

Abbreviations: $\mathrm{SC}=$ moderate/severe carditis; $\mathrm{MNC}=\mathrm{Mild} /$ no carditis $; \mathrm{C}=$ control 
After acute phase reactants decreased and the treatment ended, we compared all patients without making any distinction as moderate/severe carditis or mild/no carditis, and found statistically significant differences between all strain parameters before and after the treatment $(\mathrm{p}<0.05)$. When paired samples T-test was performed after the treatment, statistically significant increases were also found in LVGLS, LVGLSR, LVGCSR values of the patients with moderate/severe carditis and LVGLS, LVGCS, LVGCSR, RVGLS, RVGLSR values of the patients with mild/no carditis (Table 3 ).

Table 3. Strain parameters of the patients before and after the treatment

\begin{tabular}{lllll}
\hline & Pre-treatment & $\begin{array}{l}\text { Post-treatment } \\
\text { (mean } \pm \text { SD) }\end{array}$ & $p$ \\
$\begin{array}{l}\text { Moderate/severe } \\
\text { Carditis }\end{array}$ & LVGLS & $18.8 \pm 1.9$ & $22 \pm 1.4$ & .007 \\
& LVGLSR & $0.3 \pm 0.2$ & $0.62 \pm 0.27$ & .007 \\
& LVGCS & $17.9 \pm 2.5$ & $21.5 \pm 3.9$ & .025 \\
& LVGCSR & $0.43 \pm 0.26$ & $0.57 \pm 0.23$ & .111 \\
& RVGLS & $18.2 \pm 2.9$ & $20 \pm 4.4$ & .209 \\
& RVGLSR & $0.38 \pm 0.2$ & $0.55 \pm 0.3$ & .086 \\
Mild/no carditis & LVGLS & $18.5 \pm 2.7$ & $21.7 \pm 3.1$ & .001 \\
& LVGLSR & $0.38 \pm 0.18$ & $0.51 \pm 0.38$ & .194 \\
& LVGCS & $18.7 \pm 2.9$ & $22.8 \pm 3.1$ & .001 \\
& LVGCSR & $0.33 \pm 0.22$ & $0.64 \pm 0.4$ & .027 \\
& RVGLS & $19.8 \pm 3.1$ & $23.7 \pm 3.6$ & .000 \\
& RVGLSR & $0.28 \pm 0.14$ & $0.44 \pm 0.16$ & .005 \\
\hline
\end{tabular}

When we compared the strain parameters of the patients with those of the controls after the treatment, there were not any significant differences (Table 4).

Table 4. Strain parameters after the treatment.

\begin{tabular}{|c|c|c|c|c|c|c|c|}
\hline & $\begin{array}{l}\text { Moderate/st } \\
\text { Carditis (n } \\
=12)\end{array}$ & $\begin{array}{l}\text { raild } / \text { no } \\
\text { carditis ( } \mathrm{n}= \\
15)\end{array}$ & $\begin{array}{l}\text { Control (n } \\
=27)\end{array}$ & $P 1$ & $P^{2}$ & P3 & $P_{4}$ \\
\hline LVGLS & $22 \pm 1.4$ & $21.76 \pm 3.1$ & $22.1 \pm 3.5$ & .933 & .998 & .981 & .991 \\
\hline LVGLSR & $0.62 \pm 0.27$ & $0.51 \pm 0.38$ & $0.6 \pm 0.4$ & .572 & .999 & .707 & .794 \\
\hline LVGCS & $21.5 \pm 3.9$ & $22.8 \pm 3.1$ & $21.1 \pm 3.2$ & .307 & .993 & .314 & .762 \\
\hline LVGCSR & $0.57 \pm 0.23$ & $0.64 \pm 0.41$ & $0.68 \pm 0.35$ & .695 & .644 & .979 & .919 \\
\hline RVGLS & $20 \pm 4.4$ & $23.7 \pm 3.6$ & $23.9 \pm 6.8$ & .148 & .189 & .999 & .109 \\
\hline RVGLSR & $0.55 \pm 0.3$ & $0.44 \pm 0.16$ & $0.7 \pm 0.42$ & .066 & .594 & .052 & .714 \\
\hline
\end{tabular}

P1: SC/MNC/C, P2: SC/C, P3: MNC/C, P4: SC/MNC

Abbreviations: $\mathrm{SC}=$ moderate/severe carditis; $\mathrm{MNC}=\mathrm{Mild} /$ no carditis; $\mathrm{C}=$ control

\section{Discussion}

Rheumatic heart disease is one of the major causes of cardiac mortality and morbidity in school age children specifically in developing countries. The estimates show 15 million cases worldwide, 233.000 of them dying every year [12]. Therefore, the diagnosis, the treatment and the pathophysiology of the disease is of utmost importance.

Although some investigators suggest that RHD always presents as pancarditis, it is generally accepted as a disease of the endocardium and the valves, and it rarely involves the myocardium [13]. Clinical findings 
such as tachycardia and tachypnea indicate heart failure for which valvular regurgitation is the main reason, although myocardial involvement can also contribute to that process $[14,15]$. Narula et al. found no myocyte necrosis, but non-specific changes were frequently observed in the right ventricle biopsy specimens [3]. Several recent studies questioned myocardial involvement contributing to myocardial dysfunction and they proposed that RHD is only a disease of the endocardium and that myocardial influences are due to valve regurgitation $[16,17]$. For patients with rheumatic heart disease, the postmortem microscopic examination of the cardiac muscle showed lymphocyte infiltration during acute stage and fibrosis during chronic stage and functional disorders of the heart muscle were reported due to these changes [18, 19]. Myocardial biopsies demonstrated mononuclear cells and fibrin degeneration while myocardial necrosis was not reported [3]. Cross reactivity between cardiac myosin and group A beta hemolytic Streptococcal M protein has also been demonstrated [19].

Two dimensional STE can detect myocardial contraction abnormalities long before decreases in left ventricle ejection fraction are observed [20,21]. Moreover, STE is less angle dependent than Doppler based methods [21]. In this study, patients were analyzed before and after the treatment and compared to healthy controls by STE.

In the present study, significantly reduced left ventricle global longitudinal and circumferential strain and strain rates, and right ventricle global longitudinal strain and strain rates were found in patients with preserved left ventricular ejection fraction and fractional shortening in acute phase of RHD compared to healthy controls. Our results showed no statistically significant correlation with strain and strain rates and the severity of the valvular involvement. This made us think that decreases in strain were related to effects on myocardial tissue than heart failure. Supporting this view, Ozdemir et al. found higher Troponin T levels in 28 patients with active rheumatic carditis compared to healthy controls and they commend that all patients have minimal myocardial involvement especially near the endocardium by the spread of the inflammation in the connective tissue [2]. We also found recovery in left ventricle global longitudinal and circumferential and right ventricle global longitudinal strain and strain rates after treatment in patients when we used paired samples $\mathrm{T}$ test. After the treatment, all strain and strain rates increased.

In the literature, there are a limited number of studies analysing rheumatic heart disease and myocardial strain. In one of such studies, in adults with rheumatic mitral stenosis, left ventricular global longitudinal and global circumferential strain values were lower than those of the controls and recovery was seen after balloon mitral valvuloplasty. This was not due to myopathic processes but due to a tethering effect caused by the secondary restriction of the basal myocardium from mitral stenosis [22]. In another study similar to this one, 60 adult patients with rheumatic mitral stenosis having preserved ejection fraction left ventricle global longitudinal strain and strain rate values were low [23]. In a rare study by Beaton et. al. analyzing the correlation between myocardial strain and RHD in children, in latent RHD patients with normal systolic functions, left ventricle global longitudinal strain values were found to be lower than those of the controls and myocardial strain was thought to play a role in understanding the pathophysiology of latent rheumatic heart disease [24]. In this study, similar to ours, a pediatric patient group without systolic dysfunction was analyzed and the only difference was the inclusion of patients in the study during the latent stage. In our study, different from this study, global longitudinal strain values were different than those of the healthy controls only during the acute stage. This difference disappeared after the treatment, the treatment also resulted in an increase in right ventricular strain values, the difference with healthy children during the acute stage disappeared after the treatment. In pediatric patients with latent rheumatic heart disease, normal heart muscle function was demonstrated with conventional methods; a new technique, 3D strain echocardiography found normal strain values during latent stage supporting our study [25].

\section{Conclusion}

The aim of this study is to identify subtle ventricular dysfunction by speckle tracking echocardiography in children with active rheumatic heart disease, who are shown to have normal cardiac functions by conventional methods. In the literature, there are a very limited number of studies investigating myocardial strain. This is the first study assessing myocardial deformation during the acute phase of rheumatic heart disease. In 
this study, myocardial strain and strain rate values were lower in patients with acute RHD compared to healthy controls even in those with no valvular involvement. This data suggested that any patient with acute RHD can have some degree of myocardial involvement. This was thought to be due to myocardial dysfunction than valvular involvement. We need to perform more detailed studies to understand the reason behind myocardial dysfunction; however, based on available information this is more likely due to myocardial inflammation than myocardial necrosis. We need to identify the role of myocardial strain and strain rate on RHD pathophysiology, diagnosis and treatment with studies conducted on larger patient groups.

\section{Study Limitations}

While it contributes to the literature, our study has certain limitations.. First of all, the number of patients is rather low to draw a meaningful conclusion. Furthermore, we measured strain parameters only globally; radial strain was not analyzed and the 17-segment model recommended by American Heart Association was not used. Therefore, a segmental strain imaging would be preferable. Moreover, we do not have long term follow-up results.

\section{REFERENCES}

1. Narula, J., Y. Chandrasekhar, and S. Rahimtoola, Diagnosis of active rheumatic carditis: the echoes of change. Circulation, 1999.100(14): p. 1576-1581. 2. Ozdemir, O., et al., Cardiac troponin T in children with acute rheumatic carditis. Pediatr Cardiol, 2011. 32(1): p. 55-8. 3. Narula, J., et al., Does endomyocardial biopsy aid in the diagnosis of active rheumatic carditis? Circulation, 1993.88(5): p. 2198-2205. 4. Gorcsan, J. and H. Tanaka, Echocardiographic assessment of myocardial strain. Journal of the American College of Cardiology, 2011.58(14): p. 1401-1413. 5. Hasanain Ali Hameed Bshiebish a, Ali Hussein Al-Musawi a, Safaa Ali Khudeir Role of global longitudinal strain in assessment of left ventricular systolic function in patients with heart failure with preserved ejection fraction.6. Xu, T.Y., et al., Left ventricular deformation in relation to the geometric pattern in hypertensive patients. Medicine (Baltimore), 2019. 98(4): p. e14257. 7. Nikdoust, F., et al., Early diagnosis of cardiac involvement in systemic lupus erythematosus via global longitudinal strain (GLS) by speckle tracking echocardiography. J Cardiovasc Thorac Res, 2018.10(4): p. 231-235. 8. Amedro, P., et al., Speckle-Tracking Echocardiography in Children With Duchenne Muscular Dystrophy: A Prospective Multicenter Controlled Cross-Sectional Study. J Am Soc Echocardiogr, 2019. 9. Çetin, İ.İ., et al., The efficacy and safety of naproxen in acute rheumatic fever: The comparative results of 11-year experience with acetylsalicylic acid and naproxen. The Turkish journal of pediatrics, 2016. 58(5): p. 473. 10. Gewitz, M.H., et al., Revision of the Jones Criteria for the diagnosis of acute rheumatic fever in the era of Doppler echocardiography: a scientific statement from the American Heart Association. Circulation, 2015. 131(20): p. 1806-18. 11. Allen, H.D., et al., Moss 63 Adams' heart disease in infants, children, and adolescents: including the fetus and young adult. 2013: Lippincott Williams \& Wilkins. 12. Hoke, M.D.S.T.R., The worldwide epidemiology of acute rheumatic fever and rheumatic heart disease. 2011. 13. Mishra, T., et al., Myocardial dysfunction in rheumatic carditis-does it really exist? Japi, 2007. 55. 14. Edwards, B.S. and J.E. Edwards, Congestive heart failure in rheumatic carditis: Valvular or myocardial origin? 1993, Journal of the American College of Cardiology. 15. Gentles, T.L., et al., Left ventricular mechanics during and after acute rheumatic fever: contractile dysfunction is closely related to valve regurgitation. Journal of the American College of Cardiology, 2001. 37(1): p. 201-207. 16. Markowitz, M. and L. Gordis, Rheumatic fever. 1972. 17. Tandon, R., Rheumatic fever pathogenesis: Approach in research needs change. Annals of Pediatric cardiology, 2012. 5(2): p. 169. 18. Farrukh Hussain a, Tarek S. Kashour, Ivan Barac, Forgotten but not extinct: Lessons in hemodynamics and imaging from pancardiac rheumatic heart disease.19. Chopra, P. and H. Gulwani, Pathology and pathogenesis of rheumatic heart disease. Indian journal of pathology \& microbiology, 2007. 50(4): p. 685-697. 20. Escher, F., et al., New echocardiographic findings correlate with intramyocardial inflammation in endomyocardial biopsies of patients with acute myocarditis and inflammatory cardiomyopathy. Mediators of inflammation, 2013. 2013. 21. Geyer, H., et al., Assessment of myocardial mechanics using speckle tracking echocardiography: fundamentals and clinical applications. Journal of the American Society of Echocardiography, 2010. 23(4): p. 351-369. 22. Sengupta, S.P., et al., Effects of percutaneous balloon mitral valvuloplasty on left ventricular deformation in patients with 
isolated severe mitral stenosis: a speckle-tracking strain echocardiographic study. Journal of the American Society of Echocardiography, 2014.27(6): p. 639-647. 23. Ozdemir, A.O., et al., Prediction of subclinical left ventricular dysfunction with longitudinal two-dimensional strain and strain rate imaging in patients with mitral stenosis. The International Journal of Cardiovascular Imaging, 2010. 26(4): p. 397-404. 24. Beaton, A., et al., Cardiac strain findings in children with latent rheumatic heart disease detected by echocardiographic screening.Cardiol Young, 2017. 27(6): p. 1180-1185. 25. ·, R.S.M.S.G.A.H.I.M.Y.A.E.R.N.A.N.B.O.I., Subtle Myocardial Dysfunction and Fibrosis in Children with Rheumatic Heart Disease: Insight from 3D Echocardiography, 3D Speckle Tracking and Cardiac Magnetic Resonance Imaging. 2019. 